Article

\title{
Convolutional neural networks with deep supervised feature learning for remote sensing scene classification
}

\author{
Grigorios Tsagkatakis ${ }^{1}\left(\mathbb{C}\right.$ and Panagiotis Tsakalides ${ }^{1,2}$ (1)* \\ 1 Institute of Computer Science, Foundation for Research and Technology-Hellas (FORTH), 70013 Crete, \\ Greece; stivakt@ics.forth.gr (R.S.); greg@ics.forth.gr (G.T.); tsakalid@ics.forth.gr (P.T.) \\ 2 Computer Science Department, University of Crete, 70013 Crete, Greece \\ * Correspondence: greg@ics.forth.gr; Tel.: +302811392725
}

\begin{abstract}
State-of-the-art remote sensing scene classification methods employ different Convolutional Neural Network architectures for achieving very high classification performance. A trait shared by the majority of these methods is that the class associated with each example is ascertained by examining the activations of the last fully connected layer, and the networks are trained to minimize the cross-entropy between predictions extracted from this layer and ground-truth annotations. In this work, we extend this paradigm by introducing an additional output branch which maps the inputs to low dimensional representations, effectively extracting additional feature representations of the inputs. The proposed model imposes additional distance constrains on these representations with respect to identified class representatives, in addition to the traditional categorical cross-entropy between predictions and ground-truth. By extending the typical cross-entropy loss function with a distance learning function, our proposed approach achieves significant gains across a wide set of benchmark datasets in terms of classification, while providing additional evidence related to class membership and classification confidence.
\end{abstract}

Keywords: Scene classification; Deep Learning; Convolutional Neural Networks; Feature learning

\section{Introduction}

Classification of satellite and aerial imagery is a quintessential problem in remote sensing observation analysis. Although the variation in appearance and environmental conditions make this a very challenging problem, in the past few years, machine learning methods have led to a dramatic leap in performance [1,2]. In supervised image classification, both for generic imagery and remote sensing scenes, the current gold standard involves designing an appropriate Convolutional Neural Network (CNN) and training the network so that the cross-entropy loss between the predicted and the ground-truth labels is minimized. The cross-entropy is defined as the KL-divergence between the ground truth labels, encoded as a binary vector where all but one element are zero (one-hot encoding), and the predicted class label distribution from the network, obtained from the last layer after appropriate scaling via a soft-max activation function [3]. Although different loss functions have been proposed for non-classification tasks like focal loss in object detection [4] and mean-squared-error for image enhancement [5], the majority of state-of-the-art CNN architectures employ cross-entropy as the loss function to be minimized for scenarios like supervised image classification [6].

While training a CNN based on the accuracy of the predictions, quantified by the cross-entropy, is a valid and very successful approach, it fails to consider the characteristics of the features extracted by the network. Although feature extraction is an integral part of the CNN classification pipeline, unlike previous state-of-the-art methods that decouple feature extraction and classification, typically, 
the outputs of an intermediate layer, relatively close to the output layer, are considered as the features and the final fully connected layer produces the classification results [7]. As such, while end-to-end training of $\mathrm{CNNs}$, using variants of gradient descent, target the minimization of the the classification error, a wealth of information encoded in features extracted from the last fully connected layer, by large, remains untapped.

Motivated by the power of these "features" to encode useful information, in this work we extend the typically $\mathrm{CNN}$ architectural pipeline by proposing the addition of an additional output branch that generates low dimensional embedded features extracted from a given input. The proposed scheme, called CNN with Supervised Feature Learning (CNN-SFL) introduces additional terms in the typical loss function which capture the assumption that features extracted from images from the same class when appropriately embedded in a low-dimensional space, must be clustered together. In other words, the feature representation of an example should be close to a representative from the same class and far away from representatives of different classes. As such, the main contributions of this paper are summarized as follows:

- We propose the CNN-SFL, a universally-applicable scheme that can extend and enhance traditional CNN architectures.

- We demonstrate that the proposed scheme achieves better between class separation compared to traditional approaches by employing distances to class representatives.

- We provide experimental evidence that the proposed architecture surpasses, in terms of prediction accuracy, existing state-of-the-art methods, through an evaluation on three benchmark datasets.

- Our analysis also indicates that the proposed scheme is able to provide significantly more separable representations between different classes compared to more traditional approaches.

The remainder of the paper is organized as follows. In Section 2, we outline the state-of-the-art in deep learning based remote sensing image classification, focusing on research closely related to the proposed work. In section 3 we present in detail the proposed CNN-SFL method, while in Section 4 we report the experimental results and comparisons with state-of-the-art methods. The paper concludes in Section 5.

\section{Related work}

Scene classification represents a critical problem in the domain of remote sensing and numerous approaches have been proposed. In the past five years, the majority of methods considered for this problem employ the deep learning framework and most typically rely on Convolutional Neural Network architectures $[1,8]$, employing categorical cross-entropy for the final class prediction. The idea of introducing the relationship between features in addition to the cross-entropy was considered in [9] where the authors introduced a distance metric loss between the features extracted in the one-before-last layer of a $\mathrm{CNN}$ architecture. The core idea of this work is that in addition to minimizing the classification error, the network should also produce features that exhibit low inter-class and high intra-class distances.

Kang et. al [10] recently proposed the augmentation of the typical cross-entropy loss function with an additional term encoding the distances between features extracted through the Scalable Neighborhood Components Analysis (SCNA) method. The SCNA is an extension of the neighborhood component analysis, a supervised dimensionality reduction method that seeks to learn a metric space such that the leave-one-out KNN score is maximized. In [11], the authors utilized the features extracted from the last convolutional block layer of a $\mathrm{CNN}$ as input to a random forest classifier for remote sensing scene classification. In [12], the authors proposed diversity-promoting deep structural metric learning for remote sensing scene classification. Distance metric learning using CNN was also employed in [13] for remote sensing image retrieval where the similarity between a query image and the image dataset is evaluated on the features extracted from a CNN. 
In the past few years, different distance metrics have been proposed in the context of Deep Metric Learning [14]. Contrastive loss [15] aims at minimizing the distance between pairs of examples from the same class and maximize the distance between pairs of examples from different classes through the introduction of the appropriate loss function terms using Siamese network architectures. The triplet loss [16] considers triples which consist of a query example, an example from the same class, and an example from a different class. The objective in this case is to minimize the distance between examples from the same class while maximizing the distance between examples from different classes. In [17], K. Sohn proposed the N-pair loss which extended the triplet loss by considering N-1 negative examples simultaneously. The angular loss [18] considers the cosine similarity between examples, and offers scale and rotation invariance.

\section{The Convolutional Neural Network with Supervised Feature Learning (CNN-SFL) framework}

\subsection{Overall CNN-SFL design}

The proposed CNN-SFL is a universal extension of typical CNN architectures for image classification where instead of a single output, the network provides two outputs, one corresponding to the one-hot vector encoding the class predictions, and another providing the low-dimensional features. The key idea behind the CNN-SFL framework, is that traditional CNN architectures seeking to minimize the disparity between predicted and ground-truth classes, can be augmented with an additional constraint enforcing similarity between the feature representations of each example and the associated feature space of representatives corresponding to the same and to different classes. An illustrative block diagram of the propose CNN-SFL framework is shown in Figure 1 which showcases the three components of the composite loss function, namely $\mathcal{L}_{1}, \mathcal{L}_{2}, \mathcal{L}_{3}$ representing classification error, distances with between examples and same class representative, and distance to different class representative, respectively.

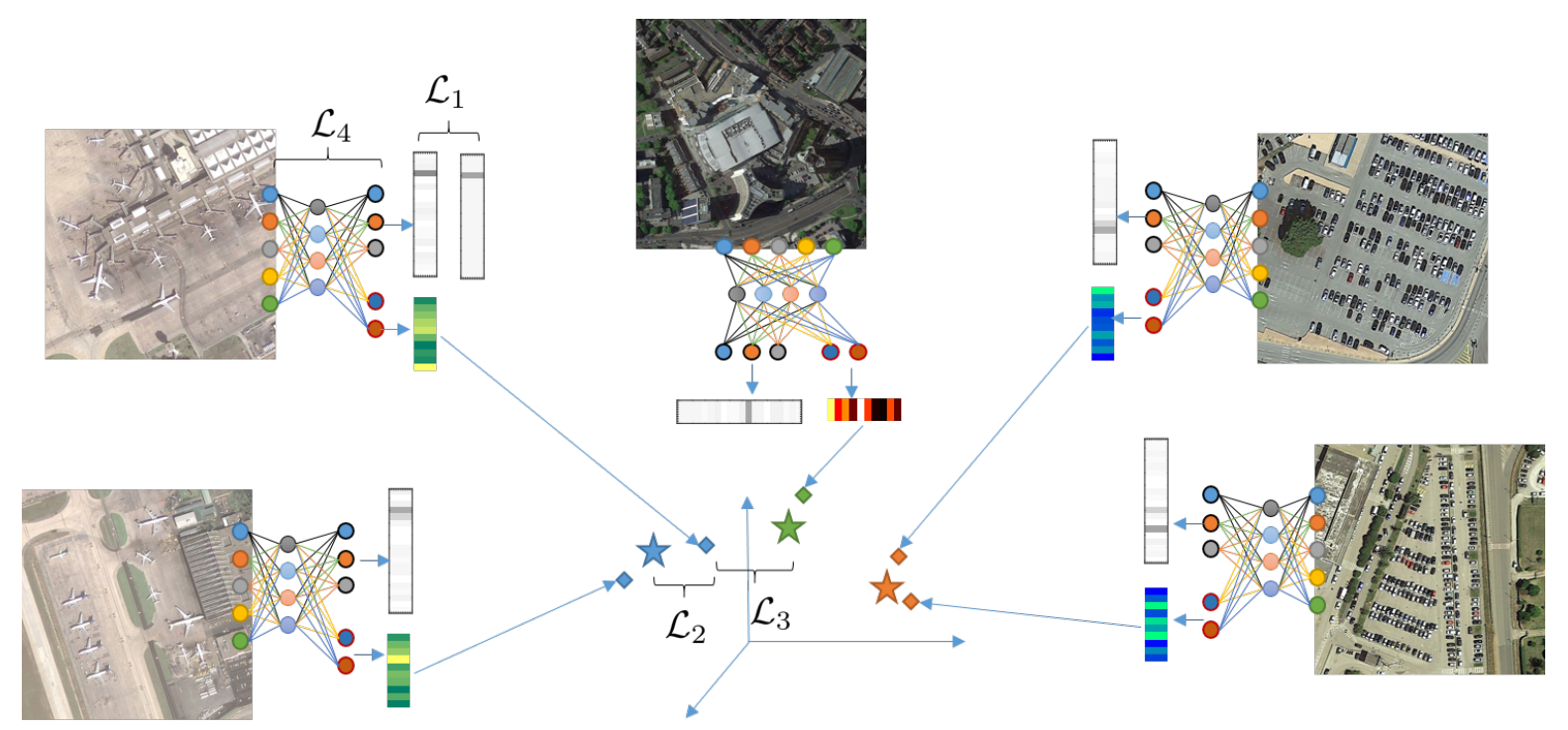

Figure 1. Block diagram of the CNN-SLF where the three loss function modules are shown. Specially, the $\mathcal{L}_{1}$ penalizes the difference between predicted and ground-truth annotation, $\mathcal{L}_{2}$ promotes small distances between feature-space representation of examples (diamonds) and representatives (stars) from the same class while $\mathcal{L}_{3}$ forces greater distances between representations and representatives from different classes. 


\subsection{CNN-SFL network architecture}

Let $\mathbf{X}=\left\{\mathbf{x}_{i} \mid i=1,2, \cdots, N\right\}$ be a set of $N$ training examples and $\mathbf{Y}=\left\{\mathbf{y}_{i} \mid i=1,2, \cdots, N\right\}$ the corresponding classes associated with each example. In our model, we assume that labels are encoded in a one-hot representation such that each label $\mathbf{y}_{i} \in \mathbb{R}^{c}$ is a vector of $c$ elements, equal to the number of classes, has every elements equal to 0 except the one associated with the class of example $x_{i}$ which is equal to 1. Furthermore, for each example, we associate the features extracted from the network which are given by $\mathbf{Z}=\left\{\mathbf{z}_{i} \mid i=1,2, \cdots, N\right\}$ and by $\mathbf{C}=\left\{\mathbf{c}_{i} \mid i=1,2, \cdots, c\right\}$ the representatives of the feature representation of each class. In addition to the representatives for each class, we also define

Let $L$ be the number of layers in the base $\mathrm{CNN}$ architecture, i.e., the backbone network. Highly successful architectures which can be utilized as base networks include the ResNet [19] and the Inception [20] architectures. In all these reference architectures, the network consists of $L-1$ convolutional blocks while the $L^{\text {th }}$ layer is a fully connected (or dense) layer with elements equal to the number of classes. For the specific case of the ResNet, the network consists of a series of convolutional blocks where the input goes through two paths. One path simply propagates the input, while the other consists of a convolution, a batch normalization, a ReLU activation, another convolution and a batch normalization. At the end of the block, these two path are added together and the output goes though a last ReLU activation. For the case of the Inception, each convolutional block consists of multiple independent paths where are all concatenated to product the block output. Exemplary paths include cascade of convolution with different kernel sizes, e.g., $1 \times 1$ followed by a $3 \times 3$ and then by a another $3 \times 3$, or max pooling layer followed by a $1 \times 1$ convolution.

For both ResNet and Inception, as well as many other state-of-the-art methods, once the convolutional blocks are calculated, the output is flattened and passed to a fully connected layer where the final layer nodes equal the number of disc int classes. The proposed architecture design, adds an additional fully connected layer $\hat{L}$ after the $(L-1)^{t h}$ convolutional block, such that the network is now equipped with two outputs, $L$ and $L$. While the $L$ output of the network is the same as with other applications and is responsible for producing class probabilities, the $L$ is responsible for the feature embedding process which is utilized by the proposed CNN-SFL in order to estimate the required distances to class representatives.

\subsection{CNN-SFL loss function}

The objective of the proposed scheme is to minimize the composite loss function $\mathcal{L}$ given by

$$
\mathcal{L}=\mathcal{L}_{0}+\lambda_{1} \mathcal{L}_{1}+\lambda_{2} \mathcal{L}_{2}
$$

where $\mathcal{L}_{1}, \mathcal{L}_{2}$ and $\mathcal{L}_{3}$ are the three components of the proposed loss functions, while $\lambda_{1}$ and $\lambda_{2}$ are scaling factors controlling the importance of each component. The first component of the loss function, $\mathcal{L}_{1}$, is the typical cross-entropy between predicted and truth class and is given by

$$
\mathcal{L}_{0}=\sum_{i=1}^{N} L\left(\mathbf{x}_{i}\right) \log \mathbf{y}_{i}
$$

which is minimized when $L\left(\mathbf{x}_{i}\right)=\mathbf{y}_{i}$, i.e., when the predictions, encoded in a one-hot encoding, thus representing probabilities associated with each class, are in agreement with the ones in the ground truth annotations.

The other two components of the composite loss functions are responsible for encoding the distance between examples and the representatives from the same class for the case of $\mathcal{L}_{2}$ and from a different class in $\mathcal{L}_{3}$. Formally, for each example we define the positive representative as the representative associated with the class that the example belongs to, and as negative representative, the representative that belongs to a different class. As such, the requirement for $\mathcal{L}_{2}$ is to minimize the 
distance between each example and its positive representative, and for $\mathcal{L}_{3}$ to maximize the distance between each example and its negative representative.

In order to enforce a small distance between the feature representation of a given example $i$ and the corresponding representation of the same class representative, the positive representative, the $\mathcal{L}_{2}$ loss is given by

$$
\mathcal{L}_{1}=\sum_{i=1}^{N} \exp \left(\left\|\hat{L}\left(\mathbf{x}_{i}\right)-\mathbf{c}_{i}\right\|_{2}^{2}\right)-1
$$

where $\mathbf{c}_{i}$ is the representative of the features belonging to the same class with example $i$. The additional one is introduced so that the minimum of the loss function approaches zero as the feature representations and the class representatives come closer.

Correspondingly, the $\mathcal{L}_{3}$ loss is minimized when the feature representation of an example and the feature representations of the representative from a different class is maximized and is given by

$$
\mathcal{L}_{2}=\sum_{i} \exp \left(\frac{-\left\|\hat{L}\left(\mathbf{x}_{i}\right)-\mathbf{c}_{\neq i}\right\|_{2}^{2}}{\tau}\right)
$$

where $\mathbf{c}_{\neq i}$ is the closest feature-space representative belonging to a different than the one of example $i$. The parameter $\tau$ is called the temperature and controlling the impact that the distance has on the value of the loss function term. In both cases, the similarities between feature space examples and the corresponding same and different class representatives are modeled through a radial basis function kernel in order to exploit the properties that the output values of this comparing two vectors is bounded between 0 and 1 .

\subsection{Class representative selection}

In order to select a representative for each class in the feature space, one can consider two scenarios. In the first scenario, the representative corresponds to a "novel" example which is generated by taking the mean of the same class examples in the feature space and is given by:

$$
\mathbf{c}_{i}=\frac{1}{N_{c}} \sum_{i=1}^{N_{c}} \hat{L}\left(\mathbf{x}_{i}\right)=\frac{1}{N_{c}} \sum_{i=1}^{N_{c}} z_{i}
$$

where $N_{c}$ is the number of examples which belong to class $c$. Another option is to select one of the training examples as the representative by solving the following minimization problem:

$$
\mathbf{c}_{i}=\min \sum_{i=1}^{N_{c}} \sum_{j=1, j \neq i}^{N_{c}}\left\|\hat{L}\left(\mathbf{x}_{i}\right)-\hat{L}\left(\mathbf{x}_{j}\right)\right\|_{2}^{2}
$$

In addition to the selection of the representative for each class, equally important is the selection of the corresponding negative representative, which is used in Eq. 4. Again, two scenarios can be considered, depending on whether the same negative representative is selected for all the examples in the class, or independent for each example. In the first scenario, the negative representative is the same for all the examples in the same class and corresponds to the representative that is closest to the positive representative for these examples, but from another class. Specifically, once the class representatives are estimated, the negative representative as found by solving:

$$
\mathbf{c}_{\neq i}=\min \sum_{j=1, j \neq i}^{N_{c}}\left\|\hat{L}\left(\mathbf{x}_{i}\right)-\hat{L}\left(\mathbf{x}_{j}\right)\right\|_{2}^{2}
$$

In the second scenario, the negative representative is estimate for each example independently. The benefit associated with the scenario is that the $\mathcal{L}_{3}$ loss is adjusted for each particular example, 
handling the case that some example from a certain class may be closer to a particular negative representative while others may be closes to another negative representative. In this case, the class representatives are estimated by solving:

$$
\mathbf{c}_{\neq i}=\min \sum_{j=1, j \neq i}^{N_{c}}\left\|\hat{L}\left(\mathbf{x}_{i}\right)-\hat{L}\left(\mathbf{x}_{j}\right)\right\|_{2}^{2}
$$

In this work, we consider the scenarios where class representatives are estimated and not selected, i.e, we consider Eq. 5 and Eq. 7. We opted for this approach since in the feature space, the estimated representatives capture more realistically the class centroid and are independent of the particular characteristics of the training set.

\subsection{Connection to distance metric learning}

In the distance metric learning framework, the objective is to estimate a matrix $M$ such that the distance between examples from the same class $\mathbf{x}_{i}, \mathbf{x}_{j}$ is smaller compared to the distance between examples from different classes $\mathbf{x}_{i}, \mathbf{x}_{k}$ where the distance is computed as the Mahalanobis distance given by

$$
\operatorname{dist}_{\mathbf{M}}\left(\mathbf{x}_{i}, \mathbf{x}_{j}\right)=\left(\mathbf{x}_{i}-\mathbf{x}_{j}\right)^{T} \mathbf{M}\left(\mathbf{x}_{i}-\mathbf{x}_{j}\right) .
$$

In order to the metric to be a proper distance, the matrix $\mathbf{M}$ must be positive semi-definite, i.e., all singular values must be XXX. When the matrix entries are real-values, matrix $\mathbf{M}$ can be decomposed using the $X X$ decomposition such that $\mathbf{M}=\mathbf{Q Q}^{T}$ where $\mathbf{Q}$ is a $\mathbf{X X}$ matrix [21]. Given this decomposition, the distance in Eq. 9 can be equivalently expressed as

$$
\operatorname{dist}_{\mathbf{M}}\left(\mathbf{x}_{i}, \mathbf{x}_{j}\right)=\operatorname{dist}_{\mathbf{Q}}\left(\mathbf{x}_{i}, \mathbf{x}_{j}\right)=\left(\mathbf{x}_{i}-\mathbf{x}_{j}\right)^{T} \mathbf{Q}^{T} \mathbf{Q}\left(\mathbf{x}_{i}-\mathbf{x}_{j}\right)=\left\|\mathbf{Q} \mathbf{x}_{i}-\mathbf{Q} \mathbf{x}_{j}\right\|_{2}^{2} .
$$

In the proposed CNN-SFL, effectively, the feature mapping layer $\hat{L}$ perform an equivalent function as the matrix $\mathbf{Q}$, albeit employing a non-linear mapping, instead of the linear mapping case when $\mathbf{Q}$ is employed. As a results, the new distance considered by the CNN-SLF is given by

$$
\operatorname{dist}_{\hat{F}}=\left\|K\left(\hat{F}\left(\mathbf{x}_{i}\right)\right)-K\left(\hat{F}\left(\mathbf{x}_{j}\right)\right)\right\|_{2}^{2} .
$$

where $K$ is the induced kernel associated with the radial basis function used in Eq. 3 and Eq. 4.

\subsection{Comparison with existing approaches}

Our work diverges from the approaches proposed in [9] in several ways. First, the proposed scheme can be regarded as an instance of multi-task learning where both a one-hot encoded vector and a feature vector are produced by the network. As such, it offers the flexibility of introducing another distinct path towards the feature space, not necessarily constrained by the given architecture. Second, we extend the idea of imposing constraints on the distances, but instead of considering the distances between examples within a batch, we consider distances between class representatives in feature space, extracted across batches, thus providing greater generalization capabilities. Last, unlike different variance of Deep Metric Learning where the objective is to maximize the similarity between examples from the same class and is thus applicable to retrieval-type settings, the proposed scheme can naturally extent state-of-the-art cross-entropy based classification approaches with the distance learning functionality, thus offering the best of both worlds.

\subsection{CNN-SFL training}

During training of a $\mathrm{CNN}$, training examples are split into batches and the backpropagation algorithm is executed using the gradient of the loss function computed for each batch (stochastic gradient descent). The complete pass over all available batches, corresponding to the entire training 
set, is called an epoch. The proposed CNN-SFL operated in two stages. For each batch, the error of the predicted classes with respect to the ground-truth classes, as well as the distances between the feature space representations and the class representatives are computed and the weights are appropriately updated. This is achieved by computing the gradient of the composite loss as the weighted sum of the individual loss functions. Once all batches have been considered, i.e. after the end of the epoch, the class representatives are re-estimated given the new updated features space mapping. In Algorithm 1, we outline the training process of the CNN-SFL.

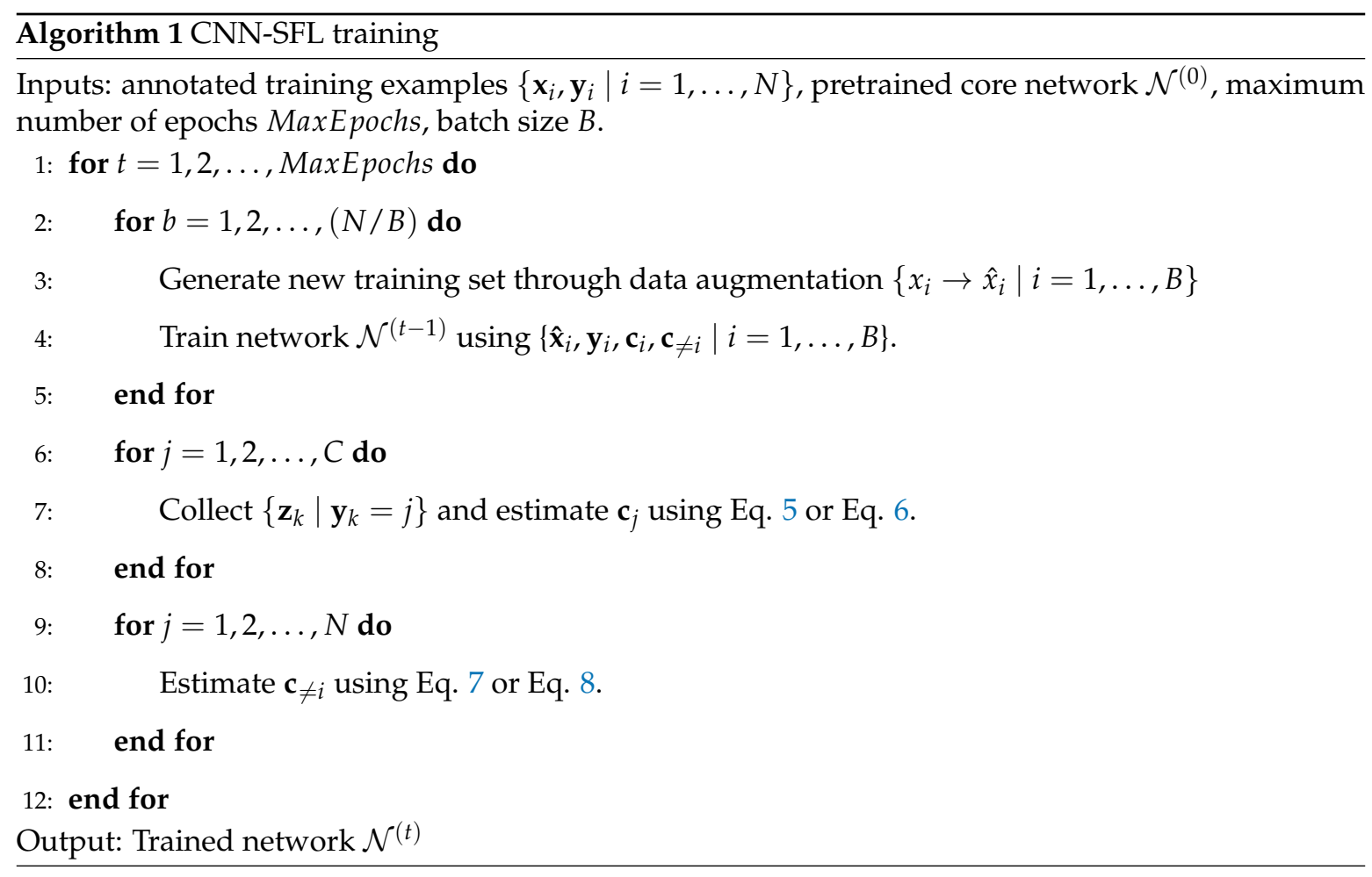

\section{Experimental Analysis}

\subsection{Datasets and evaluation metrics}

In order to understand the behavior of the proposed CNN-SFE scheme, an extensive set of experiments is considered on three representative datasets of aerial imagery, namely the UC Merced [22], the AID [23] and the NWPU-RESISC45 [2].

The UC Merced dataset ${ }^{1}$ contains aerial color (RGB) images of size $256 \times 256$ with spatial resolution $0.3 \mathrm{~m}$ from 21 land-use classes including agricultural, airplane, baseball diamond, beach, buildings, chaparral, dense residential, forest, freeway, golf course, harbor, intersection, medium density residential, mobile home park, overpass, parking lot, river, runway, sparse residential, storage tanks, and tennis courts. For each class, the datasets provides a set of 100 images.

The Aerial Image Dataset (AID) ${ }^{2}$ is a large scale collection of $10000600 \times 600$ pixel color images with resolution between 0.5 to $8 \mathrm{~m}$. The images are examples from 30 classes including airport, bare land, baseball field, beach, bridge, center, church, commercial, dense residential, desert, farmland, forest, industrial, meadow, medium residential, mountain, park, parking, playground, pond, port, 
railway station, resort, river, school, sparse residential, square, stadium, storage tanks, and viaduct. The number of images for each class varies between 220 and 420 .

The NWPU-RESISC45 datase $^{3}$ contains $31500256 \times 256$ pixel color images at spatial resolution varying between 0.2 to 30 meters. Images at classified into 45 different classes such that each class contains 100 images, while the classes include airplane, airport, baseball diamond, basketball court, beach, bridge, chaparral, church, circular farmland, cloud, commercial area, dense residential, desert, forest, freeway, golf course, ground track field, harbor, industrial area, intersection, island, lake, meadow, medium residential, mobile home park, mountain, overpass, palace, parking lot, railway, railway station, rectangular farmland, river, roundabout, runway, sea ice, ship, snowberg, sparse residential, stadium, storage tank, tennis court, terrace, thermal power station, and wetland.

In the following subsections we investigate the impact that different design choices have on the system's performance. To that end, we consider images from the AID dataset and a realistic scenario where only $5 \%$ of the dataset is available for training, in order to better emphasize the impact of different parameters on the network.

\subsection{Impact of feature dimensions}

A first aspect we explore regarding the proposed CNN-SLF model is the quantification of the impact that different sizes of the feature space representation have in term of prediction accuracy. Figure 2 presents the accuracy on the validation set achieved by the baseline ResNet101 model, and the CNN-SFL with ResNet101 as the core network and feature space dimensions equal to 32, 64 and 128.

An overall comment from the results presented in Figure 2 is that the introduction of the additional feature representation module does not reduce the classification accuracy of the standard core model. Examining the different dimensions of the feature space, the results indicate that the 128-dimensional space can have a significant impact in performance, offering gains of more than $3 \%$ compared to the baseline mode.

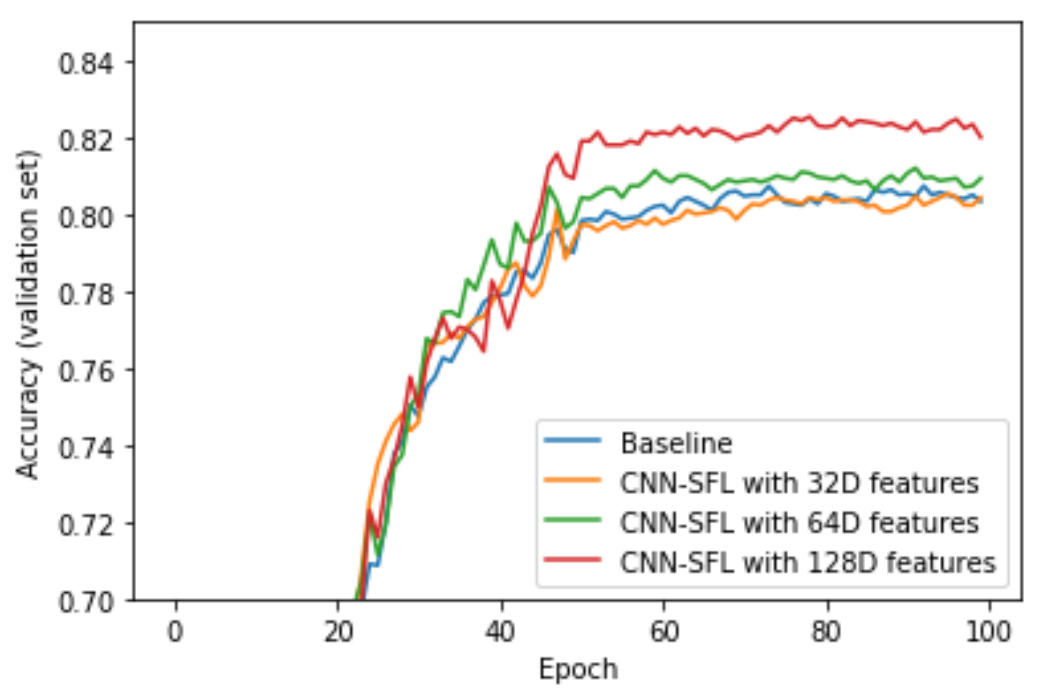

Figure 2. Validation set accuracy as a function of training epoch for the baseline and the proposed CNN-SFL method, considering the values for the feature space representation, namely 32,64 and 128 dimensional spaces.

3 http://www.escience.cn/people/JunweiHan/NWPU-RESISC45.html 


\subsection{Impact of parameter selection}

In order to get a better understanding of the performance of the proposed scheme, we explore the impact of loss function component weighting and impact of temperature parameters. Specifically, in Table 1, we explore the impact of weighting between the $\mathcal{L}_{1}$ and $\mathcal{L}_{2}$ given by parameters $\lambda_{1}$ and $\lambda_{2}$, as well as the temperature parameter $\tau$.

Regarding the impact of $\lambda_{1}$ and $\lambda_{2}$, an interesting observation is that while their impact in terms of training error is minimal, the performance gains are more pronounced for the testing/validation error. Furthermore, utilizing two loss function components, for intra and inter class similarities, leads to the best performance. Regarding the temperature parameter $\tau$, evidence suggest that their is impact in terms of performance when this value is utilized.

Table 1. Impact of loss component weighting.

\begin{tabular}{|c|c|c|c|c|}
\hline \multicolumn{3}{|c|}{ Parameter } & \multicolumn{2}{c|}{ Error } \\
\hline$\tau$ & $\lambda_{1}$ & $\lambda_{2}$ & Training & Testing \\
\hline 0.01 & 10 & 0 & 90.47 & 81.93 \\
\hline 0.01 & 0 & 10 & 90.87 & 81.98 \\
\hline 0.01 & 10 & 10 & 90.87 & 82.42 \\
\hline 0.1 & 10 & 10 & 90.87 & 82.83 \\
\hline
\end{tabular}

In order to gain a deeper understanding on the behavior of the CNN-SLF, Figure 3 visualizes the feature-based class representatives in a matrix format and the associated singular values distribution for this matrix. Ideally, one would like class representatives to be as distinct as possible, which in more mathematical way, can be revealed by quantifying the linear dependencies between them. The matrix visualization, but more importantly, the singular values shown in Figure 3 indicate that class representations from different representatives are relatively orthogonal in the 128-dimensional feature space. As a result, mapping a new test example on this feature space will lead to a representation which is much closer (in terms of projection magnitude) to the a given class representative compared to all others.

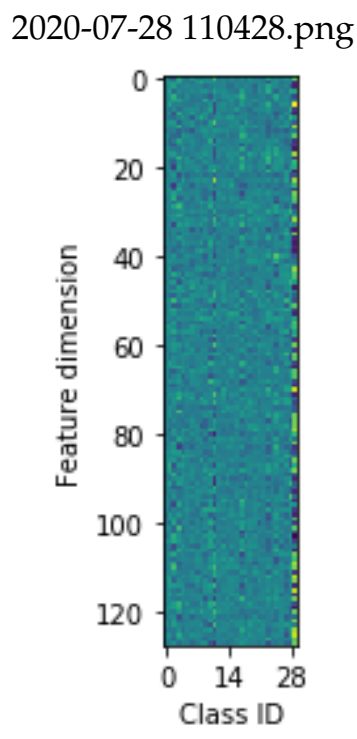

$$
\text { 2020-07-28 110418.png }
$$

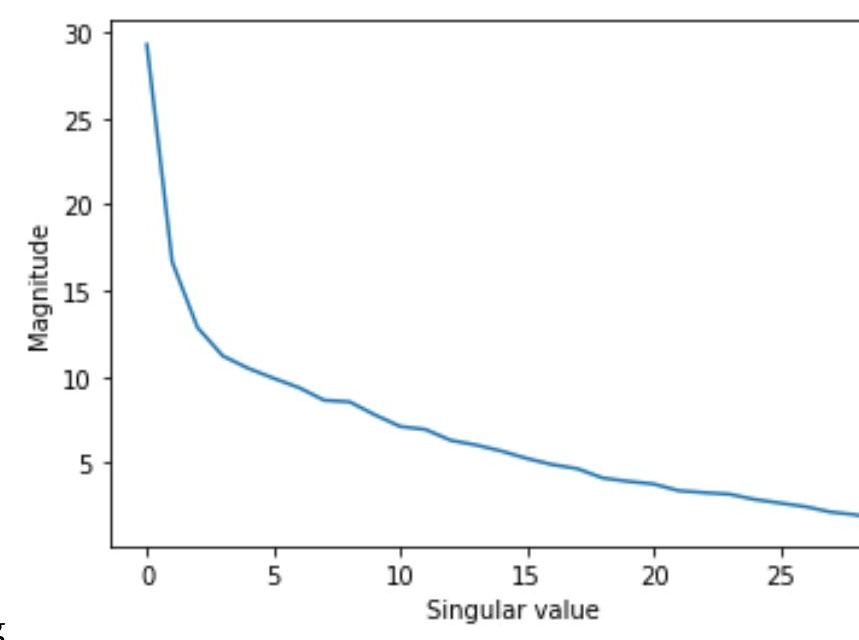

Figure 3. Visualization of feature based class representative (left) and the singular values distribution of the class representative matrix (right). 


\subsection{Visualization in low-dimensional spaces}

To better understand the impact of the feature embedding module, we employ the $t$-SNE manifold learning method [24] in order to project the extracted features into 2D spaces and allow the visualization of the outputs. For the case of the baseline network, the t-SNE maps the 2048-dimensional outputs from the first fully connected layer, which is typically considered as the feature representation, while for the CNN-SFL, the mapping is from the 128-dimensional space to the $2 D$ space for visualization. The resulting scatter plots are shown in Figure 4, the top part for the baseline model and the bottom for the proposed CNN-SLF. In addition to visualizing the extracted features, for the case of the CNN-SLF, we also plot the 128-dimensional feature representations for the class representatives (black circles).

Examining the scatter plots in Figure 4, we can clearly see that the separation between different classes achieved by the CNN-SFL is significantly between compared to the baseline model. While for the baseline model, examples from different classes are highly intermingled, for the case of the CNN-SFL, the classes are clearly separated owning to the introduction of the $\mathcal{L}_{2}$ loss function (points that are not properly clustered correspond to misclassifications). However, even for the case of samples falling away from their corresponding class region, we observe there are cases where such samples are still closer to their own class region, compared to other class regions. Furthermore, the class representatives are, as expected, centered on the region covered by examples from the corresponding class, indicating the $\mathcal{L}_{1}$ loss indeed acts as an attractor.

\subsection{Distance based class separation}

In addition to the classification of imaging using the cross-entropy loss as it is traditionally done, the proposed CNN-SFL framework is also capable of estimating distances between examples and class representatives. To quantify this behavior, Figure 5 presents histograms of the distance between samples and class representatives from the same and more different classes. Figure 5(a) present the histogram for AID with 5\% training and and Figure 5(b) for UCMD with 80\% training data.

In both cases, we observe that distances between representatives from the correct class are in general smaller compared to representatives from the wrong classes. For the case of AID, the two corresponding distributions have some overlap which is a result of misclassification, while for the case of the UCMD which achieves an almost perfect accuracy, there is no observable overlap between the two distributions.

Another observation we can make from these plots is that ideally, all the distances with the correct class representatives should be zero and all the distances with the incorrect classes should have the maximum value. However, the distributions are not delta functions but have significant volume. Even for the case where almost all examples are correctly classified, there is a portion of samples that have a non-zero distance to the correct class representative. This distance can be utilized as a proxy to the confidence associated with each prediction where smaller distances indicate a more reliable prediction. Furthermore, we imposing a threshold between the modes of the same and different class distance distributions, we can allow the network to output "unknown class" responses, unlike traditional cross-entropy-driven CNNs which will always predict a class, even if the particular examples does not belong to the available ones.

\subsection{Characteristics of misclassifications}

Figures 6, 7 and 8 present the confusion matrices for the CNN-SFL predictions on the AID, the UCMD and the RESISC45 datasets respectively. For the case of AID, the system incorrectly classifies resorts as parks, which is a reasonable mistake given that both cases are characterized by a significant amount of grassland, or in a similar spirit, confusing desert with bare land. This is also observed for the case of the UCMD dataset, where misclassifications correspond to confusing medium residential with dense residential and sparse residential. The same observation also applied to the RESISC45 dataset 


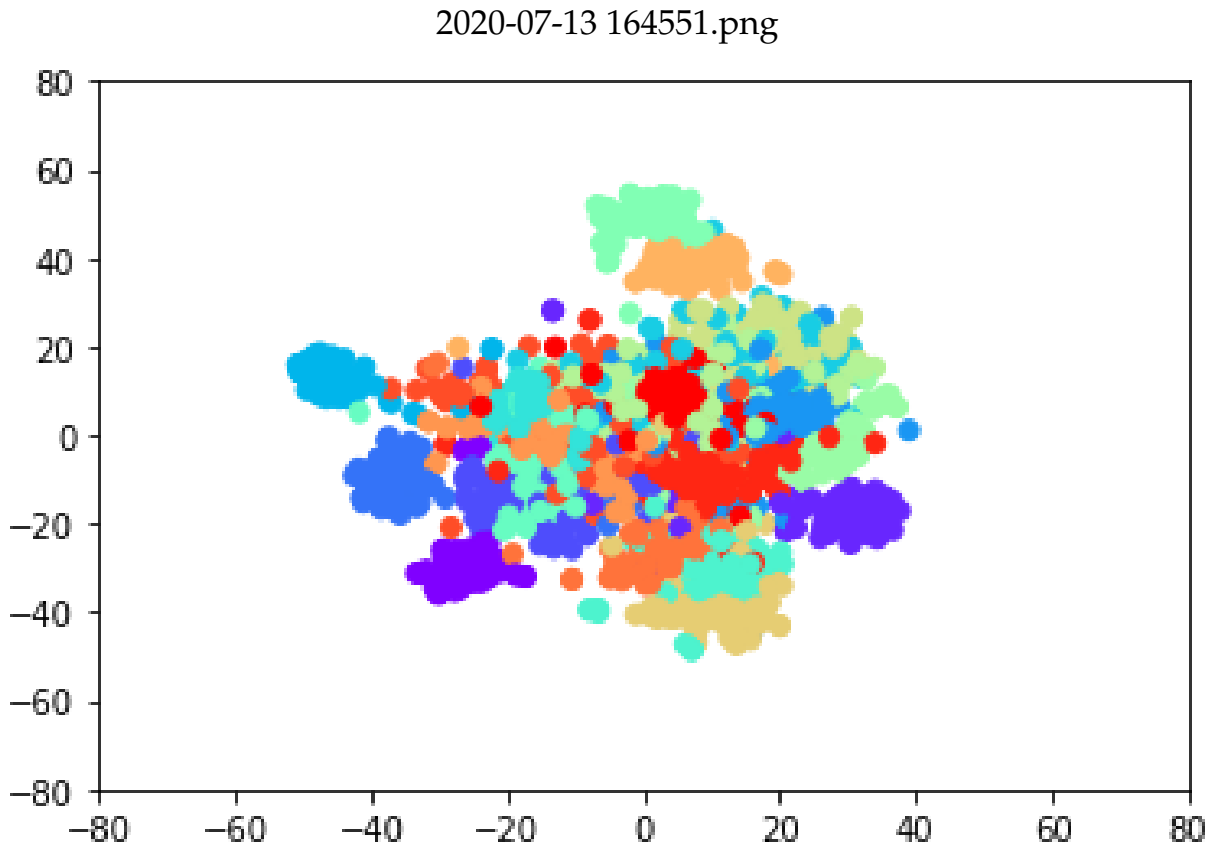

(a) Baseline model.

2020-07-13 164056.png

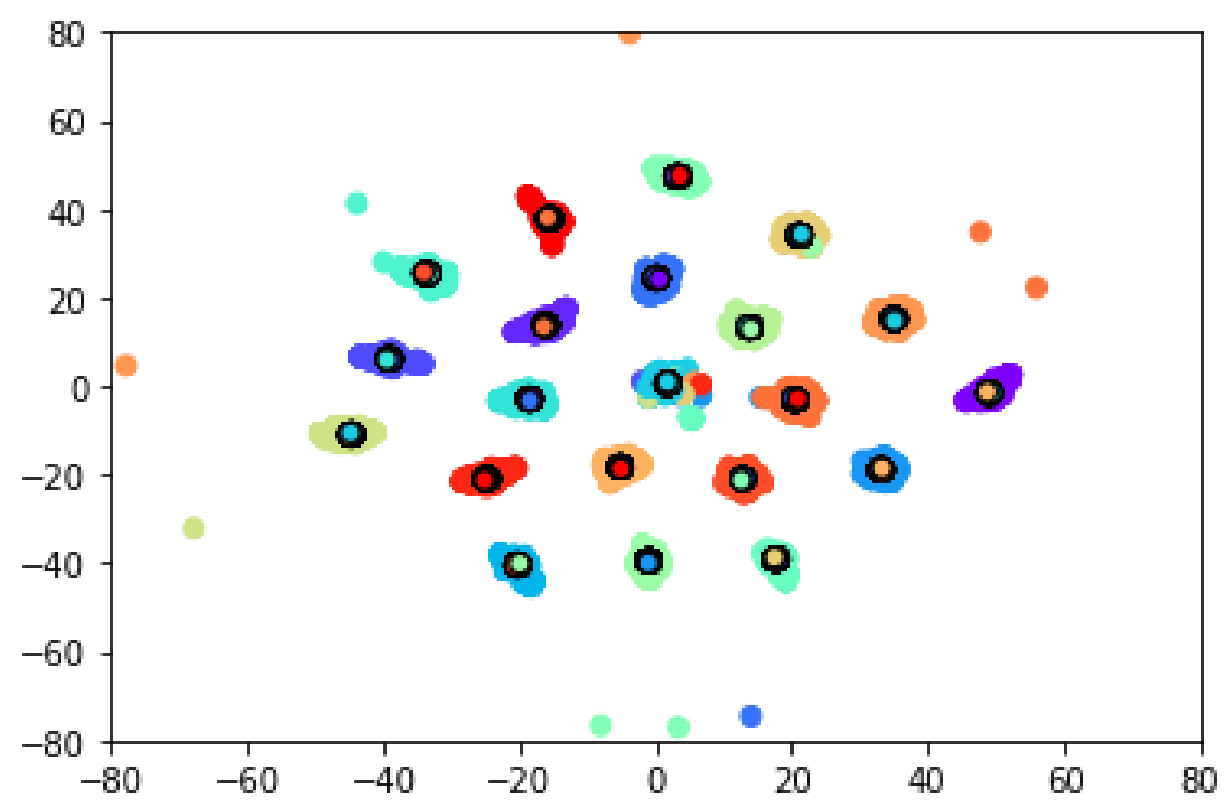

(b) CNN-SFL model.

Figure 4. 2D mapping of samples and class representatives obtained from (a) the ResNet101 baseline model and (b) the CNN-SFL model.

\subsection{Comparison with state-of-the-art}

In order to gain a better understanding of the benefits offered by the proposed CNN-SFL method, we provide a detailed comparison with current state-of-the-art methods in the benchmark dataset. Specifically, Table 2 presents the performance for the UCMD dataset, Table 3 for the AID dataset and Table 4 for the RESISC45 dataset. In all cases, we consider two training set ratio, following the reported results from previous works. In addition to our method, we also consider three highly successfully 
2020-07-15 150618.png

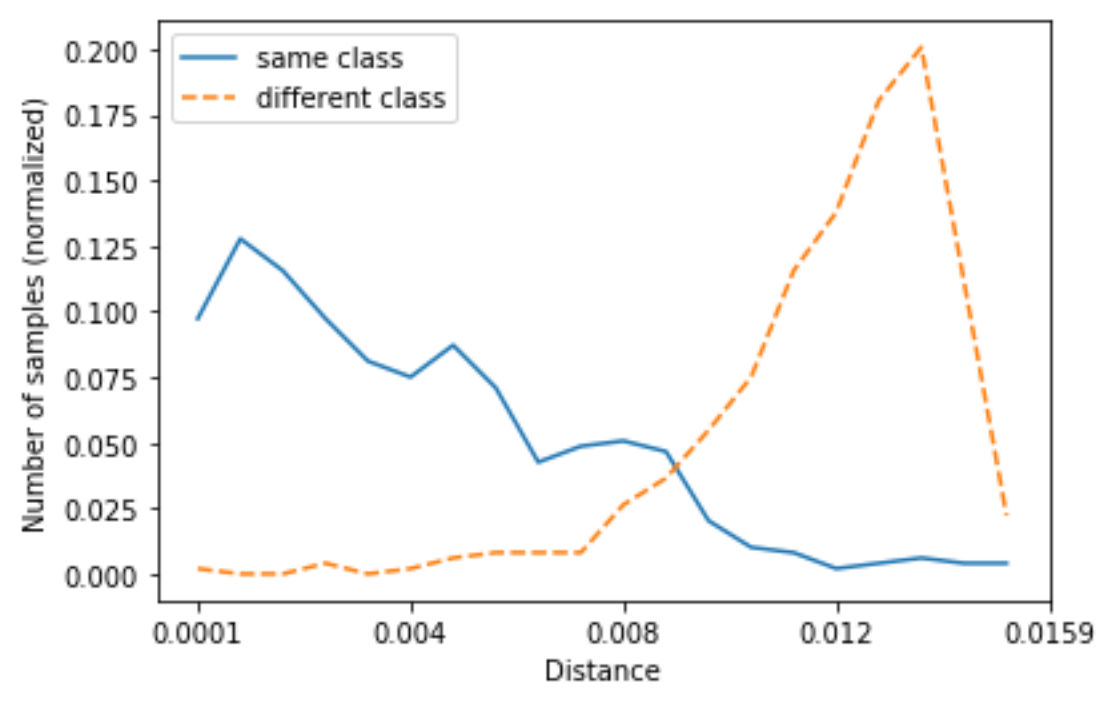

(a) AID with $5 \%$ training data.

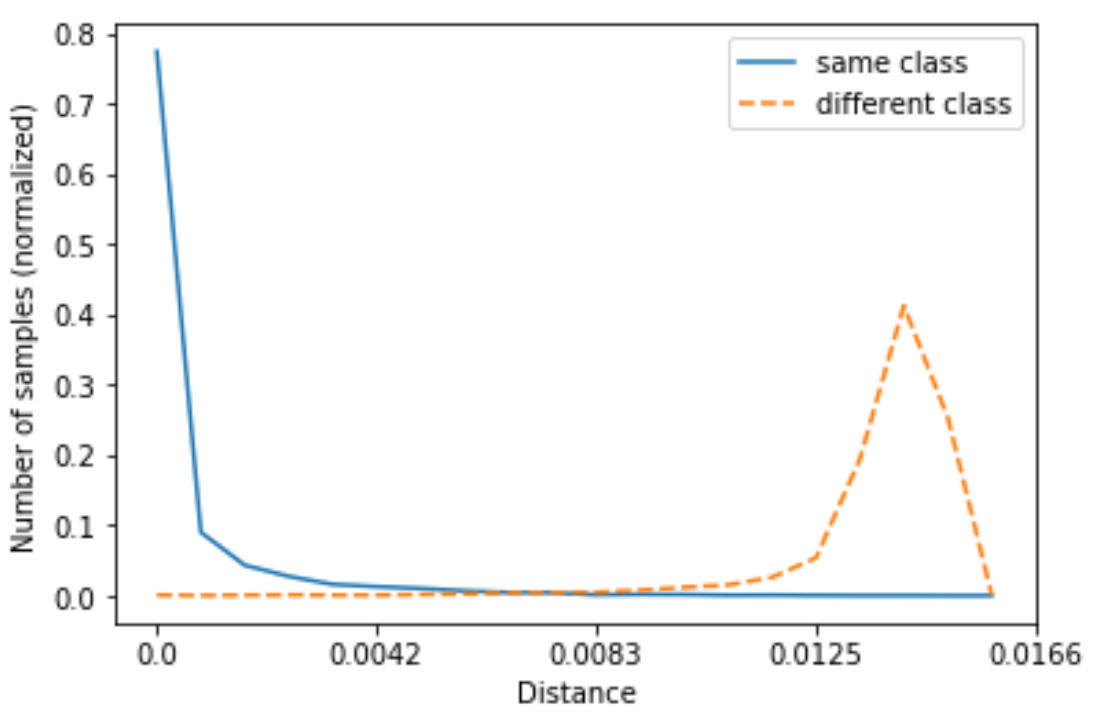

(b) UCMD with $80 \%$ training data.

Figure 5. Histogram of distances between samples and representative from the same class (' $\left.{ }^{\prime}\right)$ and from different class ('-') for AID with 5\% training data (a) and for UCMD with 80\% training data (b).

CNN architectures, namely CaffeNet [25], VGG-VD-16 [26] and GoogLeNet [27], in addition to two recently proposed methods for scene classification which also consider the notions of distances during classification, specifically, the MDFR [28] and the D-CNN [9].

Overall, in almost all datasets and training set ratio settings, the experimental results indicate that the proposed CNN-SFL achieves top performance, significantly surpassing generic $\mathrm{CNN}$ approaches and the recently presented scene-classification-focused methods. In fact in certain cases, like the UCMD dataset, the accuracy is almost perfect, so that any extremely small error can be attributed to human labelling ambiguities, i.e., medium versus dense residential. Furthermore, we observe that the performance gains are more significant in the low-training-ratio regimes, indicating that the proposed methods is able to handle more efficiently limited training sets. 


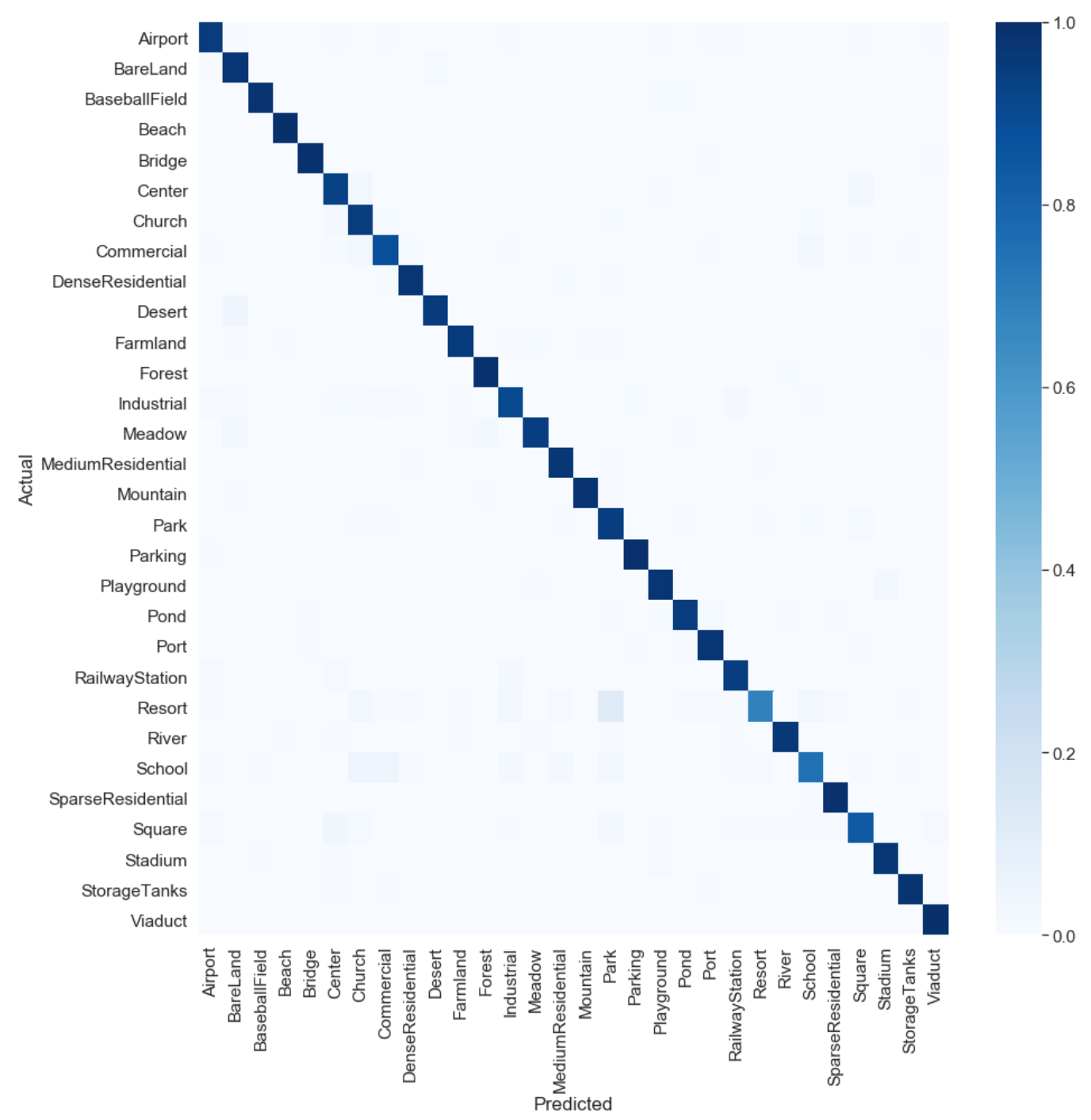

Figure 6. Confusion matrix for the AID with $50 \%$ training data.

Table 2. Classification accuracy for the UCMD dataset.

\begin{tabular}{|l|c|c|}
\hline \multirow{2}{*}{ Method } & \multicolumn{2}{|c|}{ Training ratio } \\
\cline { 2 - 3 } & $50 \%$ & $80 \%$ \\
\hline CaffeNet [23] & 93.98 & 95.02 \\
\hline VGG-VD-16 [23] & 94.14 & 95.21 \\
\hline GoogLeNet [23] & 92.70 & 94.31 \\
\hline MDFR [28] & - & 98.02 \\
\hline D-CNN [9] & - & 98.93 \\
\hline CNN-SFL (proposed) & $\mathbf{9 7 . 1 4}$ & $\mathbf{9 9 . 2 9}$ \\
\hline
\end{tabular}

\section{Conclusions}

In this work, we propose the CNN-SFL, a universally applicable extension of traditional CNN architectures which achieves very low class prediction error by incorporating both the traditional categorical cross-entropy loss with a novel feature based distance learning loss. A detailed experimental validation for remote sensing scene classification demonstrates that the proposed scheme can surpass state-of-the-art methods, while additional information related to distance to class representative can 


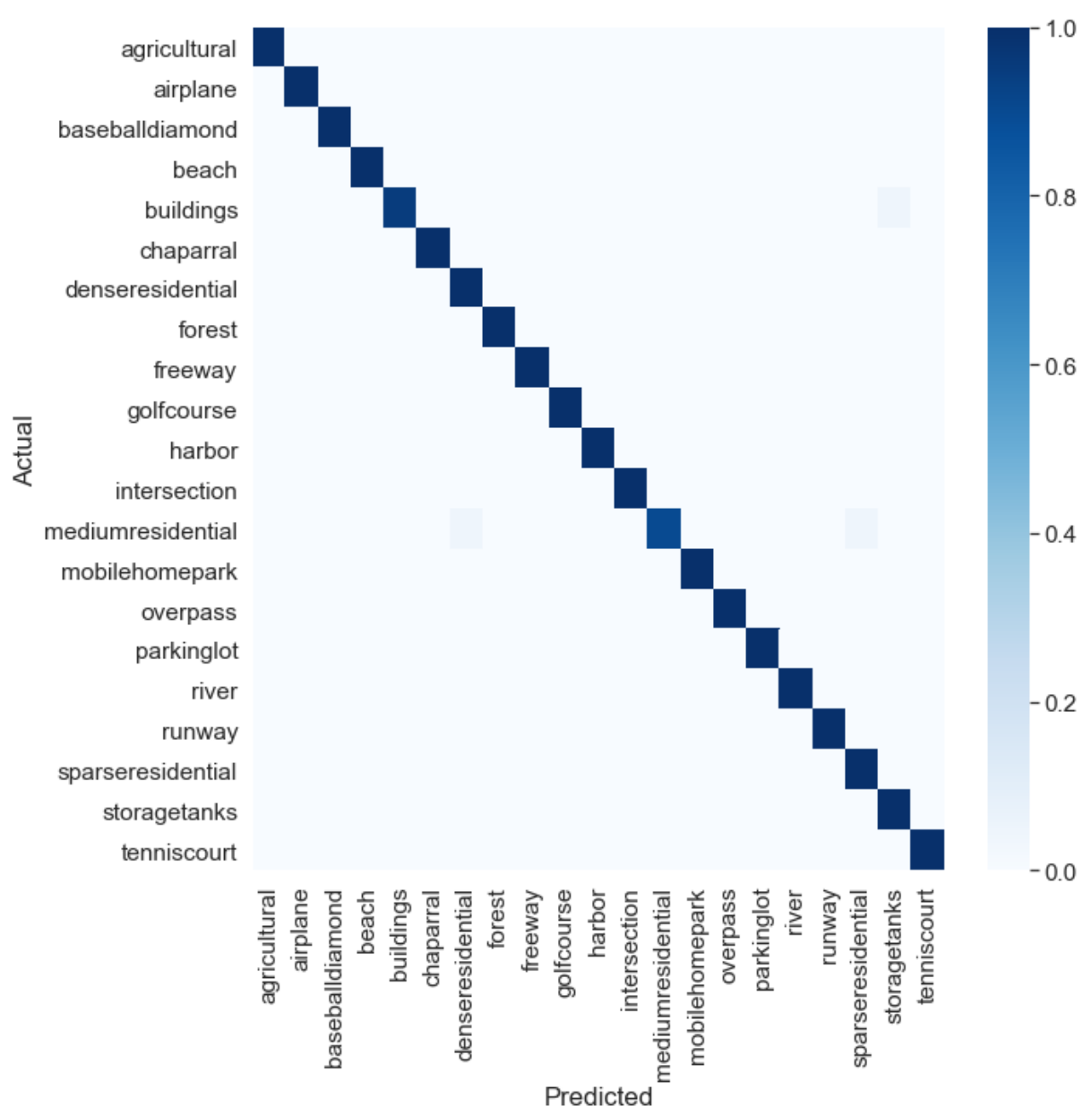

Figure 7. Confusion matrix for the UCMD with $80 \%$ training data.

Table 3. Classification accuracy for the AID dataset.

\begin{tabular}{|l|c|c|}
\hline \multirow{2}{*}{ Method } & \multicolumn{2}{|c|}{ Training ratio } \\
\cline { 2 - 3 } & $20 \%$ & $50 \%$ \\
\hline CaffeNet [23] & 86.86 & 89.53 \\
\hline VGG-VD-16 [23] & 86.59 & 89.64 \\
\hline GoogLeNet [23] & 83.44 & 86.39 \\
\hline MDFR [28] & 90.62 & 93.37 \\
\hline D-CNN [9] & 90.82 & $\mathbf{9 6 . 8 9}$ \\
\hline CNN-SFL (proposed) & $\mathbf{9 1 . 9 3}$ & 94.82 \\
\hline
\end{tabular}

Table 4. Classification accuracy for the RESISC45 dataset.

\begin{tabular}{|l|c|c|}
\hline \multirow{2}{*}{ Method } & \multicolumn{2}{|c|}{ Training ratio } \\
\cline { 2 - 3 } & $10 \%$ & $20 \%$ \\
\hline CaffeNet [23] & 78.01 & 81.08 \\
\hline GoogLeNet [2] & 82.57 & 86.02 \\
\hline MDFR [28] & 83.37 & 86.89 \\
\hline D-CNN [9] & 89.22 & 91.89 \\
\hline CNN-SFL (proposed) & $\mathbf{9 0 . 1 3}$ & $\mathbf{9 2 . 2 7}$ \\
\hline
\end{tabular}

provide insights into the quality of the prediction. The proposed scheme can be easily adapted to similar problems in remote sensing such as pixel-level classification and segmentation. 


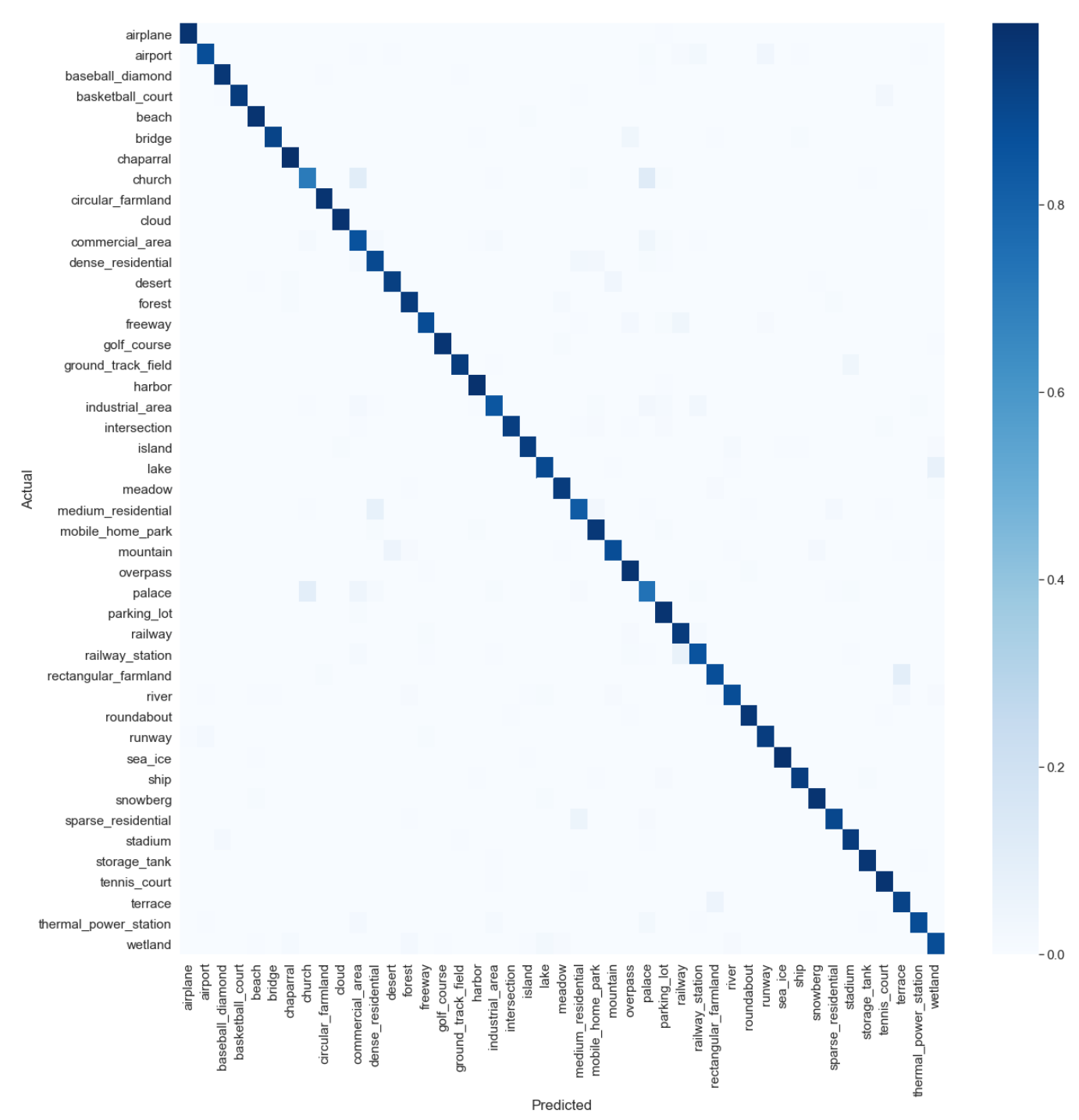

Figure 8. Confusion matrix for the RESISC45 with 20\% training data.

Author Contributions: "conceptualization, G.T.; methodology, G.T.; formal analysis, G.T.; writing-original draft preparation, G.T.; writing-review and editing, G.T., P.T..

Funding: his research work work was partially funded by the Marie Skłodowska-Curie CALCHAS project (contract no. 842560) within the H2020 Framework Program of the European Commission and by the Hellenic Foundation for Research and Innovation (HFRI) and the General Secretariat for Research and Technology (GSRT), under HFRI Faculty grant no. 1725 (V4-ICARUS).

Conflicts of Interest: “The authors declare no conflict of interest.".

\section{References}

1. Maggiori, E.; Tarabalka, Y.; Charpiat, G.; Alliez, P. Convolutional neural networks for large-scale remote-sensing image classification. IEEE Transactions on Geoscience and Remote Sensing 2016, 55, 645-657.

2. Cheng, G.; Han, J.; Lu, X. Remote sensing image scene classification: Benchmark and state of the art. Proceedings of the IEEE 2017, 105, 1865-1883.

3. Goodfellow, I.; Bengio, Y.; Courville, A. Deep Learning; MIT Press, 2016. http://www.deeplearningbook. org.

4. Lin, T.Y.; Goyal, P.; Girshick, R.; He, K.; Dollár, P. Focal loss for dense object detection. Proceedings of the IEEE international conference on computer vision, 2017, pp. 2980-2988. 
5. Tsagkatakis, G.; Aidini, A.; Fotiadou, K.; Giannopoulos, M.; Pentari, A.; Tsakalides, P. Survey of deep-learning approaches for remote sensing observation enhancement. Sensors 2019, 19, 3929.

6. Li, Y.; Zhang, H.; Xue, X.; Jiang, Y.; Shen, Q. Deep learning for remote sensing image classification: A survey. Wiley Interdisciplinary Reviews: Data Mining and Knowledge Discovery 2018, 8, e1264.

7. Gu, J.; Wang, Z.; Kuen, J.; Ma, L.; Shahroudy, A.; Shuai, B.; Liu, T.; Wang, X.; Wang, G.; Cai, J.; others. Recent advances in convolutional neural networks. Pattern Recognition 2018, 77, 354-377.

8. Zou, Q.; Ni, L.; Zhang, T.; Wang, Q. Deep learning based feature selection for remote sensing scene classification. IEEE Geoscience and Remote Sensing Letters 2015, 12, 2321-2325.

9. Cheng, G.; Yang, C.; Yao, X.; Guo, L.; Han, J. When deep learning meets metric learning: Remote sensing image scene classification via learning discriminative CNNs. IEEE transactions on geoscience and remote sensing 2018, 56, 2811-2821.

10. Kang, J.; Fernandez-Beltran, R.; Ye, Z.; Tong, X.; Ghamisi, P.; Plaza, A. Deep Metric Learning Based on Scalable Neighborhood Components for Remote Sensing Scene Characterization. IEEE Transactions on Geoscience and Remote Sensing 2020.

11. Boualleg, Y.; Farah, M.; Farah, I.R. Remote sensing scene classification using convolutional features and deep forest classifier. IEEE Geoscience and Remote Sensing Letters 2019, 16, 1944-1948.

12. Gong, Z.; Zhong, P.; Yu, Y.; Hu, W. Diversity-promoting deep structural metric learning for remote sensing scene classification. IEEE Transactions on Geoscience and Remote Sensing 2017, 56, 371-390.

13. Yun, M.S.; Nam, W.J.; Lee, S.W. Coarse-to-Fine Deep Metric Learning for Remote Sensing Image Retrieval. Remote Sensing 2020, 12, 219.

14. Kaya, M.; Bilge, H.Ş. Deep metric learning: A survey. Symmetry 2019, 11, 1066.

15. Chopra, S.; Hadsell, R.; LeCun, Y. Learning a similarity metric discriminatively, with application to face verification. 2005 IEEE Computer Society Conference on Computer Vision and Pattern Recognition (CVPR'05). IEEE, 2005, Vol. 1, pp. 539-546.

16. Schroff, F.; Kalenichenko, D.; Philbin, J. Facenet: A unified embedding for face recognition and clustering. Proceedings of the IEEE conference on computer vision and pattern recognition, 2015, pp. 815-823.

17. Sohn, K. Improved deep metric learning with multi-class n-pair loss objective. Advances in neural information processing systems, 2016, pp. 1857-1865.

18. Wang, J.; Zhou, F.; Wen, S.; Liu, X.; Lin, Y. Deep metric learning with angular loss. Proceedings of the IEEE International Conference on Computer Vision, 2017, pp. 2593-2601.

19. He, K.; Zhang, X.; Ren, S.; Sun, J. Identity mappings in deep residual networks. European conference on computer vision. Springer, 2016, pp. 630-645.

20. Szegedy, C.; Vanhoucke, V.; Ioffe, S.; Shlens, J.; Wojna, Z. Rethinking the inception architecture for computer vision. Proceedings of the IEEE conference on computer vision and pattern recognition, 2016, pp. 2818-2826.

21. Suárez, J.L.; García, S.; Herrera, F. A tutorial on distance metric learning: mathematical foundations, algorithms and software. arXiv preprint arXiv:1812.05944 2018.

22. Yang, Y.; Newsam, S. Bag-of-visual-words and spatial extensions for land-use classification. Proceedings of the 18th SIGSPATIAL international conference on advances in geographic information systems, 2010, pp. 270-279.

23. Xia, G.S.; Hu, J.; Hu, F.; Shi, B.; Bai, X.; Zhong, Y.; Zhang, L.; Lu, X. AID: A benchmark data set for performance evaluation of aerial scene classification. IEEE Transactions on Geoscience and Remote Sensing 2017, 55, 3965-3981.

24. Maaten, L.v.d.; Hinton, G. Visualizing data using t-SNE. Journal of machine learning research 2008, 9, 2579-2605.

25. Jia, Y.; Shelhamer, E.; Donahue, J.; Karayev, S.; Long, J.; Girshick, R.; Guadarrama, S.; Darrell, T. Caffe: Convolutional architecture for fast feature embedding. Proceedings of the 22nd ACM international conference on Multimedia, 2014, pp. 675-678.

26. Simonyan, K.; Zisserman, A. Very deep convolutional networks for large-scale image recognition. arXiv preprint arXiv:1409.1556 2014.

27. Szegedy, C.; Liu, W.; Jia, Y.; Sermanet, P.; Reed, S.; Anguelov, D.; Erhan, D.; Vanhoucke, V.; Rabinovich, A. Going deeper with convolutions. Proceedings of the IEEE conference on computer vision and pattern recognition, 2015, pp. 1-9. 
17 of 17

28. Zhang, J.; Zhang, M.; Shi, L.; Yan, W.; Pan, B. A Multi-Scale Approach for Remote Sensing Scene Classification Based on Feature Maps Selection and Region Representation. Remote Sensing 2019, 11, 2504. 